\title{
Patients with non-relapsing ANCA-associated vasculitis have increased numbers of circulating IL-10 producing Th17 cells
}

\author{
B Wilde ${ }^{1,2}$, M Thewissen ${ }^{1}$, P van Paassen ${ }^{1}$, M Hilhorst $^{1}$, J Damoiseaux ${ }^{1}$, O Witzke ${ }^{2}$, J W Cohen Tervaert ${ }^{\text {* }}$ \\ From 5th European Workshop on Immune-Mediated Inflammatory Diseases \\ Sitges-Barcelona, Spain. 1-3 December 2010
}

\section{Introduction/aim}

IL-17 producing T-cells (Th17) were recently defined as a new, pro-inflammatory $\mathrm{T}$-cell subset and are considered to have a key role in autoimmune diseases.

Importantly, it was recently described that anti-inflammatory regulatory T-cells (Treg) are able to convert to pro-inflammatory Th17 cells ("Plasticity") and vice versa. Little is known about the Th17 response or plasticity in ANCA-associated vasculitis (AAV). Therefore, we investigated Th17 responses in AAV.

\section{Patients and methods}

47 patients with ANCA-associated vasculitis and 12 agematched healthy controls (HC) were studied. PBMC were isolated by ficoll gradient centrifugation and stimulated for 4 hours with phorbol-myristate-acetate/ionomycin in presence of brefeldin A. Intracellular staining was performed to detect IFNg, IL-4, IL-10 and IL-17A producing T-helper-cells by flow cytometry. Ten renal biopsies with necrotizing-crescentic-glomerulonephritis (NCGN) were stained for IL-17 by immunohistochemistry.

\section{Results}

AAV patients in remission $(\mathrm{n}=27)$ and with active disease $(n=20)$ had increased numbers of circulating IL-17A ${ }^{+}$ T-helper-cells as compared to $\mathrm{HC}(2.04 \pm 1.65 \%$ vs. $0.73 \pm 0,36 \%, \mathrm{p}<0.0005$ and $1.85 \pm 2.15 \%$ vs. $0.73 \pm 0,36 \%$, $\mathrm{p}=0.05)$. Lesional $\mathrm{IL}-17^{+}$cells were present in renal biopsies with necrotizing crescentic glomerulonephritis (NCGN). Moreover, IL-10 $0^{+} / \mathrm{IL}-17^{+} \mathrm{T}$-helper-cells were found both in $\mathrm{HC}$ and AAV patients. However, AAV patients showed higher numbers of $\mathrm{IL}-10^{+} / \mathrm{IL}-17^{+}$
T-helper-cells than HC $(0.054 \pm 0.048 \%$ vs. $0.025 \pm 0.014 \%$, $\mathrm{p}<0.05)$. Furthermore, patients with non-relapsing disease course had significantly more IL-10 producing cells Th17 cells than patients with relapsing disease course $(0.063$ $\pm 0.039 \%$ vs. $0.041 \pm 0.056 \%, \mathrm{p}<0.05)$.

\section{Conclusion}

The results of this study emphasize the importance of circulating and lesional Th17 cells in AAV. IL-17 $7^{+}$ cells participate in renal inflammation related to AAV. Elevated numbers of IL-10 producing Th17-cells are demonstrated for the first time in AAV and might point at enhanced plasticity. Further efforts are needed to unravel the role of Th17 cells in AAV.

\section{Author details}

${ }^{1}$ Dept. of Internal Medicine, Division of Clinical and Experimental Immunology, University Hospital Maastricht, Maastricht, The Netherlands.

${ }^{2}$ Dept. of Nephrology, University Duisburg-Essen, Essen, Germany.

Published: 25 November 2010 